\title{
DNM1L-related mitochondrial fission defect presenting as refractory epilepsy
}

\author{
Jason R Vanstone ${ }^{1}$, Amanda M Smith ${ }^{1}$, Skye McBride ${ }^{1}$, Turaya Naas ${ }^{1}$, Martin Holcik ${ }^{1,2}$, Ghadi Antoun ${ }^{2}$, \\ Mary-Ellen Harper ${ }^{2}$, Jean Michaud ${ }^{3}$, Erick Sell ${ }^{1}$, Pranesh Chakraborty ${ }^{1}$, Martine Tetreault ${ }^{4,5}$, \\ Care4Rare Consortium ${ }^{1}$, Jacek Majewski ${ }^{4,5}$, Stephen Baird ${ }^{1}, K_{\text {Ky }}$ M Boycott ${ }^{1}$, David A Dyment ${ }^{1}$, \\ Alex MacKenzie ${ }^{1}$ and Matthew A Lines ${ }^{\star, 1}$
}

Mitochondrial fission and fusion are dynamic processes vital to mitochondrial quality control and the maintenance of cellular respiration. In dividing mitochondria, membrane scission is accomplished by a dynamin-related GTPase, DNM1L, that oligomerizes at the site of fission and constricts in a GTP-dependent manner. There is only a single previous report of DNM1L-related clinical disease: a female neonate with encephalopathy due to defective mitochondrial and peroxisomal fission (EMPF; OMIM \#614388), a lethal disorder characterized by cerebral dysgenesis, seizures, lactic acidosis, elevated very long chain fatty acids, and abnormally elongated mitochondria and peroxisomes. Here, we describe a second individual, diagnosed via whole-exome sequencing, who presented with developmental delay, refractory epilepsy, prolonged survival, and no evidence of mitochondrial or peroxisomal dysfunction on standard screening investigations in blood and urine. EEG was nonspecific, showing background slowing with frequent epileptiform activity at the frontal and central head regions. Electron microscopy of skeletal muscle showed subtle, nonspecific abnormalities of cristal organization, and confocal microscopy of patient fibroblasts showed striking hyperfusion of the mitochondrial network. A panel of further bioenergetic studies in patient fibroblasts showed no significant differences versus controls. The proband's de novo DNM1L variant, NM_012062.4:c.1085G >A; NP_036192.2:p. (Gly362Asp), falls within the middle (oligomerization) domain of DNM1L, implying a likely dominant-negative mechanism. This disorder, which presents nonspecifically and affords few diagnostic clues, can be diagnosed by means of DNM1L sequencing and/or confocal microscopy.

European Journal of Human Genetics (2016) 24, 1084-1088; doi:10.1038/ejhg.2015.243; published online 25 November 2015

\section{INTRODUCTION}

Mitochondrial dynamics, that is, the dynamic fission and fusion of portions of the mitochondrial network within cells, are essential to mitochondrial quality control in the nervous system and other tissues (reviewed in Archer $^{1}$ and Itoh et $a l^{2}$ ). During mitochondrial and peroxisomal division, outer membrane cleavage is accomplished by dynamin 1-like protein (DNM1L), a GTPase that forms multimeric collars at, and constricts, specific fission sites. ${ }^{3,4}$ Cells or mice deficient for DNM1L or its murine paralog, Drp1, exhibit giant, net-like, structurally abnormal mitochondria that assume a perinuclear distribution..$^{5,6}$ In neurons, deficiency of Drp1 prevents mitochondria from trafficking correctly to the axon and synaptic bouton, with ensuing abnormalities of synaptic vesicle trafficking. ${ }^{7-9}$ Altered mitochondrial fission, fusion, or distribution are seen in several neurodegenerative conditions, including Charcot-Marie-Tooth disease type 2A, Parkinson's disease, and autosomal dominant optic atrophy, indicating a specific structural requirement for mitochondrial fission in neurons. ${ }^{2}$

In 2007, Waterham et al. ${ }^{10}$ reported a de novo heterozygous DNM1L pathogenic variant in a female neonate with a lethal encephalopathy characterized by cerebral dysgenesis, seizures, lactic acidosis, elevated very long chain fatty acids, and abnormal mitochondrial and peroxisomal elongation. The clinical course was severe, with survival of 37 days. The sole other mention in the literature of this condition is a meeting abstract by Yoon et al. ${ }^{11}$ regarding a sibling pair with encephalopathy, hepatic dysfunction, giant intraneuronal mitochondria, and neonatal lethality, with compound heterozygous DNM1L changes consistent with recessive inheritance. Here, we describe a child with a comparatively indolent phenotype comprising epilepsy, developmental delay, normal mitochondrial and peroxisomal screening tests, and prolonged survival. Whole-exome sequencing showed the patient's phenotype to be the result of a de novo missense substitution, NM_012062.4:c.1085G > A; NP_036192.2:p.(Gly362Asp), in exon 10 of DNM1L, altering a conserved glycine residue in the middle (oligomerization) domain of DNM1L.

\section{MATERIALS AND METHODS}

Clinical subjects

All procedures were in accord with the declaration of Helsinki and informed consent was obtained from the participants before enrollment. The research protocol was approved by the Research Ethics Board of the Children's Hospital of Eastern Ontario.

${ }^{1}$ Children's Hospital of Eastern Ontario Research Institute and University of Ottawa, Ottawa, Ontario, Canada; ${ }^{2}$ Department of Biochemistry, Microbiology, and Immunology, Faculty of Medicine, University of Ottawa, Ottawa, Ontario, Canada; ${ }^{3}$ Department of Pathology and Laboratory Medicine, Children's Hospital of Eastern Ontario and University of Ottawa, Ottawa, Ontario, Canada; ${ }^{4}$ Department of Human Genetics, McGill University, Montreal, Quebec, Canada; ${ }^{5}$ McGill University and Genome Quebec Innovation Center, Montreal, Quebec, Canada

*Correspondence: Dr MA Lines, Division of Metabolics and Newborn Screening, Department of Pediatrics, Children's Hospital of Eastern Ontario, 401 Smyth Road, Ottawa, Ontario K1H 8L1, Canada. Tel: +1 $6137377600 \times 2821$; Fax: +1 613 738-4216; E-mail: mlines@cheo.on.ca

Received 16 June 2015; revised 2 October 2015; accepted 14 October 2015; published online 25 November 2015 


\section{Whole-exome sequencing}

DNA extraction, sequencing, and exome analysis were performed in the proband as previously described. ${ }^{12}$ After filtering, a total of 459 rare (minor allele frequency $<1 \%$ ) exonic and splice-site variants remained for consideration. We first inspected the data for interesting recessive (homozygous or compound heterozygous) variants, finding no promising candidates. Of 287 heterozygous rare variants in the proband, one variant in DNM1L (NM_012062.4:c.1085G > A) was selected as potentially interesting, and was confirmed to be de novo by Sanger sequencing of the proband and parents. The observed variant has been submitted to the database at http://databases.lovd.nl/ shared/variants/DNM1L (individual ID 00047314).

\section{Cell culture}

Primary patient fibroblast cultures were established based on a 2-mm sterile skin biopsy according to standard clinical protocols. Patient and healthy adult control fibroblasts were cultured in DMEM supplemented with $10 \%$ fetal bovine serum, penicillin $(100 \mathrm{U} / \mathrm{ml}) /$ streptomycin $(100 \mu \mathrm{g} / \mathrm{ml})$, and $2 \mathrm{~mm}$ glutamine (growth media) according to standard protocols. All media components were obtained from Hyclone (Logan, UT, USA).

\section{Laser scanning confocal microscopy}

Fibroblasts were incubated for 15 min with $200 \mathrm{~nm}$ MitoTracker Green FM (MTG; Life Technologies, Carlsbad, CA, USA). Live imaging of the mitochondrial network was performed on an Olympus IX81 microscope (Olympus, Center Valley, PA, USA) using FV10-ASW software (Olympus).

Alternatively, for high-throughput imaging, fibroblasts were fixed with $4 \%$ paraformaldehyde, blocked and permeabilized according to standard protocols, incubated overnight at $4{ }^{\circ} \mathrm{C}$ with a rabbit anti-TOMM20 antibody (1:1000, sc-111415; Santa Cruz Biotechnology, Dallas, TX, USA), followed by detection with an anti-rabbit Cy3 antibody (1:400, 111-165-003; Jackson ImmunoResearch Laboratories, West Grove, PA, USA). Imaging was performed on an Opera High Content Screening System (Perkin Elmer, Waltham, MA, USA) using Columbus software (Perkin Elmer).

\section{Micro-oximetry}

Mitochondrial oxygen consumption rate (OCR) and extracellular acidification rate (ECAR) were measured in fibroblasts as previously described ${ }^{13}$ using the Seahorse XF-24 Extracellular Flux Analyzer (Seahorse Biosciences, North Billerica, MA, USA). Cells were seeded at 50000 cells/well $24 \mathrm{~h}$ before the assay. OCR was measured basally and following the sequential addition of $1 \mu \mathrm{g} / \mathrm{ml}$ oligomycin, $1 \mu \mathrm{M}$ carbonyl cyanide 3-chlorophenylhydrazone (CCCP), and $1 \mu \mathrm{M}$ antimycin A with $0.5 \mu \mathrm{M}$ rotenone (all reagents were obtained from Sigma, St Louis, MO, USA). Values for OCR were corrected to non-mitochondrial OCR assessed after the addition of antimycin A and normalized to total protein.

For the assessment of glycolytic function, ECAR was measured in a starved state and following the addition of $10 \mathrm{~mm}$ glucose (Sigma). All values were corrected to non-glycolytic ECAR and normalized to total protein.

\section{Measurement of mitochondrial content and inner membrane potential}

Fibroblasts were stained with either 200 nм MTG (for assessment of mitochondrial content) or $50 \mathrm{~nm}$ tetramethylrhodamine ethyl ester perchlorate (TMRE; for assessment of mitochondrial inner membrane potential; Sigma) for $15 \mathrm{~min}$. Cells were trypsinized, resuspended in phosphate-buffered saline containing $0.2 \%$ bovine serum albumin (Sigma), and kept on ice until fluorescence was measured on a Cyan ADP 9 analyzer (Beckman Coulter, Mississauga, ON, Canada). Autofluorescence from an unstained control sample was subtracted from the mean fluorescence of the stained sample.

\section{Assessment of cell viability}

Fibroblasts were plated at $10000-15000$ cells/well in 96-well plates. The following day, growth media were replaced with fresh media containing either $25 \mathrm{~mm}$ glucose or $10 \mathrm{~mm}$ galactose (to force aerobic respiration; Sigma) as the major carbon source, as well as $100 \mathrm{~nm}$ of YOYO-1 Iodide (Life Technologies). Measurements of cell death, as indicated by YOYO-1 fluorescence, were performed over $72 \mathrm{~h}$ using the IncuCyte ZOOM Live Cell Imaging System (Essen Bioscience, Ann Arbor, MI, USA). For end point normalization, YOYO-1positive cell counts were expressed as a fraction of the total number of cells.

\section{RESULTS}

\section{Clinical findings}

The proband was born to healthy, nonconsanguineous Caucasian parents following a normal pregnancy. Delivery was at term, by Caesarian section, due to failure to progress. There were no concerns in the first months of life. The family sought medical attention at 6 months of age as the patient was not meeting expected motor milestones. Head control was attained at 6-8 months, sitting at $18-20$ months, crawling at $2 \frac{1}{2}$ years, and pulling-to-stand at 3 years of age. The proband's subsequent course has been one of profound global developmental delay, and at the last clinical assessment (age 7 years), he remained nonambulatory with the use of $<10$ monosyllabic words. There have been no episodes of regression. Epilepsy was first recognized at 1 year of age, initially with unresponsive episodes of bilateral eye deviation. He has subsequently developed myoclonic, focal, and generalized tonic clonic seizures, and experienced several episodes of status epileptics. His mother currently describes his seizures as (1) several daily episodes of immobile, unresponsive staring with eye blinking and (2) up to 100 daily episodes of head drop, mouth twitching, and eye flickering, lasting $\sim 20 \mathrm{~s}$ in duration. In general, seizures have proven refractory to medical therapy and, of multiple antiepileptic medications (valproic acid, levetiracetam, clobazam, clonazepam, topiramate, phenobarbital, piracetam) tried in varying combinations, only piracetam has been effective. EEG showed background slowing with frequent epileptiform activity in the frontal and central head regions (Supplementary Figure 1). Brain MRI and MR spectroscopy (3 occasions between 2 and 5 years) was normal. On examination, the patient was normally grown and non-dysmorphic. He exhibited mild bilateral ptosis, inverted feet (patient able to passively dorsiflex his ankles), and a crossed adductor response.

Multiple clinical genetic and metabolic investigations were undertaken in the patient. Chromosomal microarray, SCN1A sequencing, and biochemical studies (creatine kinase, lactate, plasma and CSF amino acids, acylcarnitine profile, very long chain fatty acids) were normal. Quadriceps biopsy was normal apart from the presence of concentric cristae and/or increased dense granules in some mitochondria, and many subsarcolemmal mitochondrial aggregates on electron microscopy (Figure 1). Respiratory chain enzymologies in muscle and skin fibroblasts and lactate/pyruvate ratio in fibroblasts were normal. mtDNA sequencing identified two homoplasmic LHON secondary variants, m.4216T $>C$ and m.4917A $>$ G, as well as a third, homoplasmic rare variant, $\mathrm{m} .8477 \mathrm{~T}>\mathrm{C}$, inherited from the patient's healthy mother.

To identify the cause of the patient's chronic encephalopathy, we performed whole-exome sequencing that identified a missense substitution, NM_012062.4:c.1085G > A; NP_036192.2:p.(Gly362Asp), in exon 10 of DNM1L. Segregation analysis confirmed this change to be de novo. This variant is not represented in the Exome Aggregation Consortium (ExAC) database, and the in silico algorithms SIFT and Polyphen2 predict it to be pathogenic. ${ }^{14-16}$ Primary fibroblasts from the patient exhibited a striking, hyperfused mitochondrial phenotype when compared with control primary fibroblasts (Figure 2). In order to assess mitochondrial function in further detail, a panel of bioenergetics studies was performed (micro-oximetry, flow cytometry-based assessment of mitochondrial bulk and inner membrane potential, cell survival under mandatory respiration); no difference between patient and control fibroblasts was detected (Figure 3). 

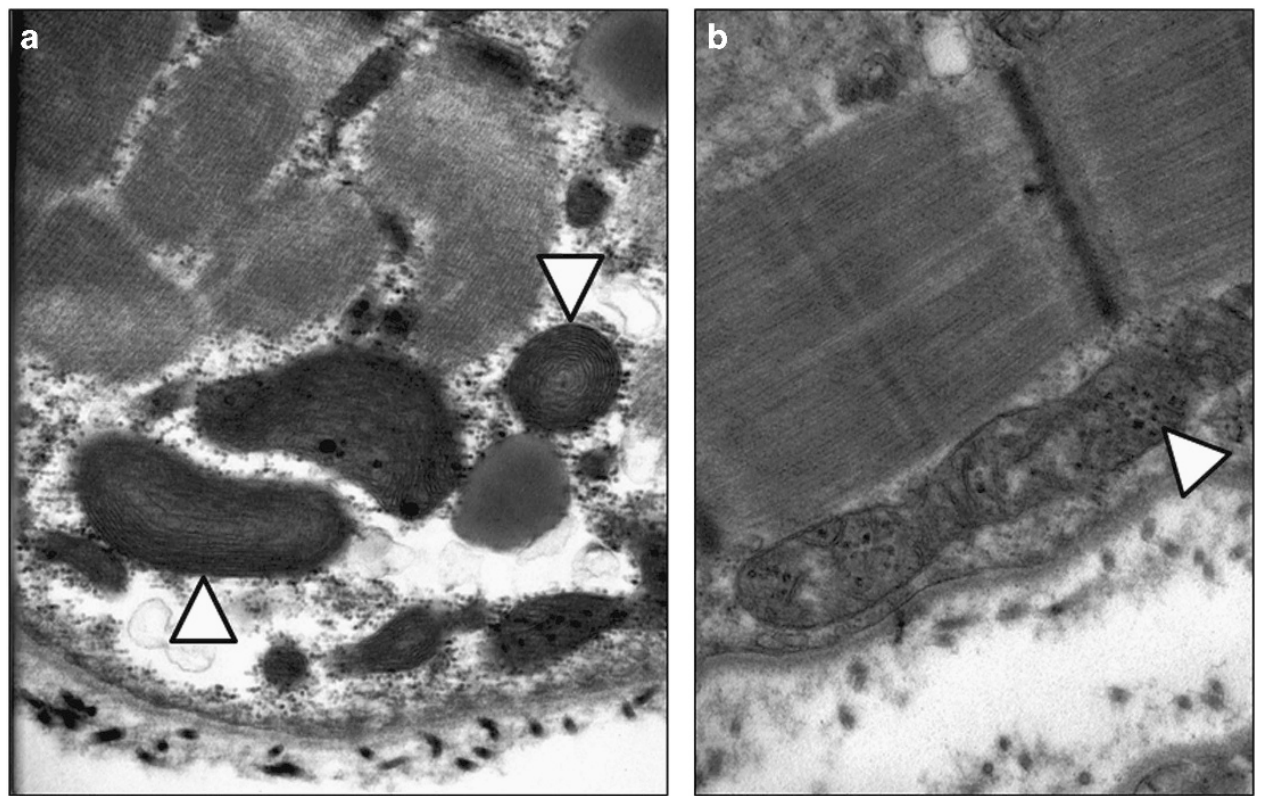

Figure 1 Electron microscopy of patient muscle indicates abnormalities in mitochondria. (a) Electron micrograph of patient muscle with arrowheads indicating mitochondria with abnormal, concentric cristae. (b) Electron micrograph of patient muscle with arrowhead indicating mitochondria with increased dark, granular inclusions.
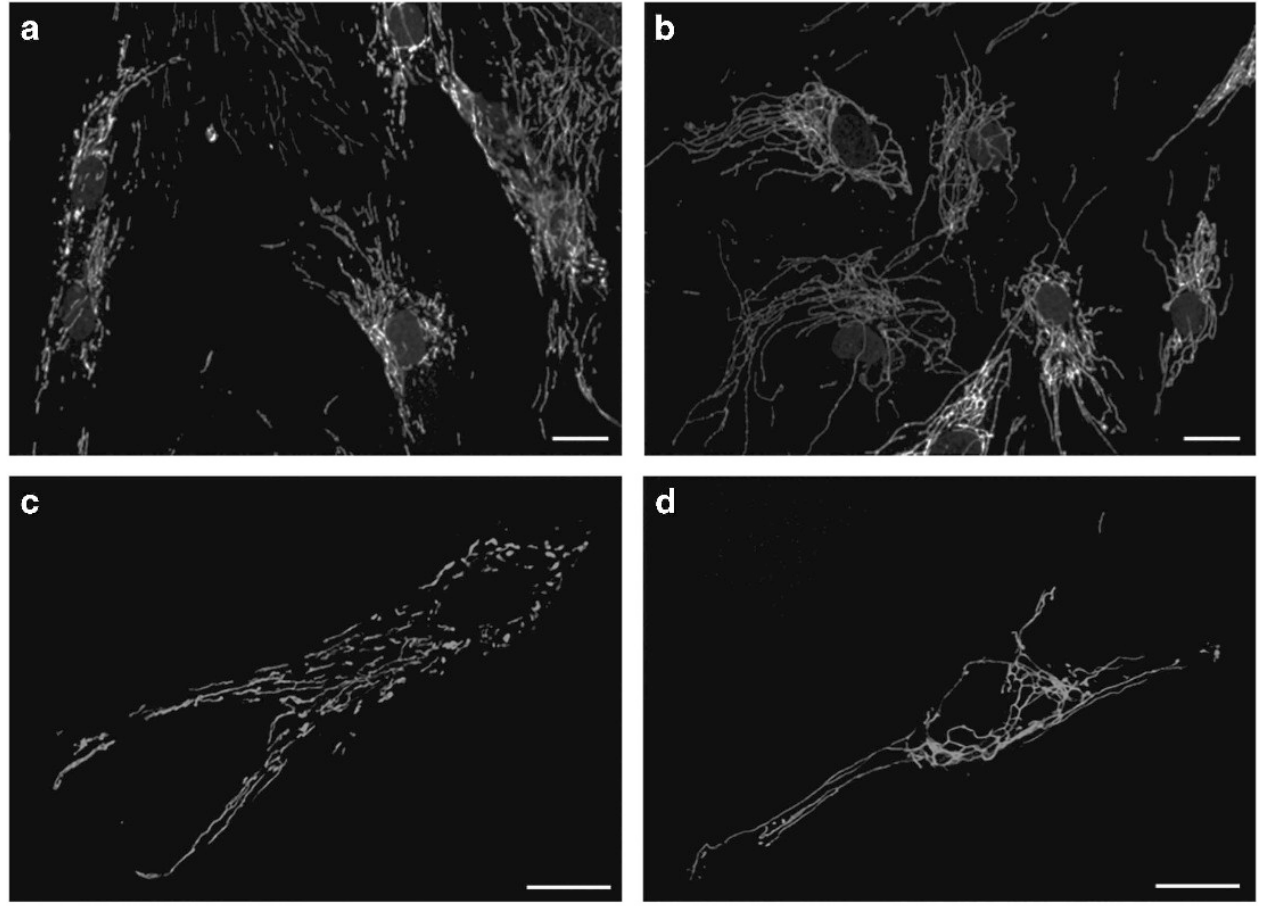

control fibroblasts

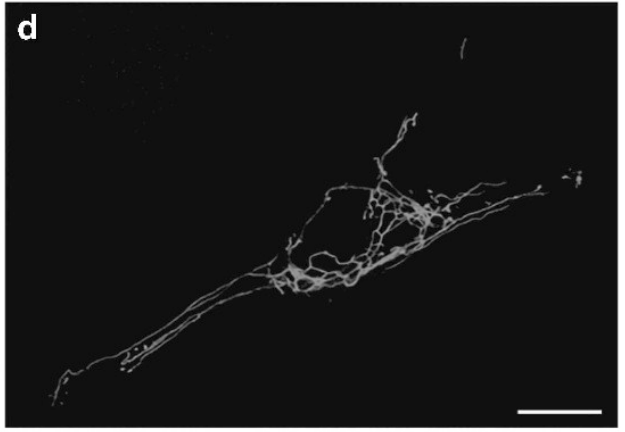

patient fibroblasts

Figure 2 Patient fibroblasts exhibit a hyperfused mitochondrial network morphology. TOMM20-stained fibroblasts from (a) control and (b) patient with hyperfused mitochondrial network. Representative single cell from live cell imaging of MitoTracker Green FM-stained (c) control and (d) patient with hyperfused mitochondrial network. Scale bar $=25 \mu \mathrm{m}$.

\section{DISCUSSION}

To our knowledge, this report constitutes the second published case of a pathogenic de novo variant in DNM1L. In contrast to the de novo, lethal, infantile-onset phenotype reported by Waterham et al. ${ }^{10}$ and the sibling pair with recessive changes referred to in the unpublished abstract by Yoon et al, ${ }^{11}$ our proband presented with prolonged survival and a relatively nonspecific refractory epilepsy, with few clinical signs suggestive of a mitochondrial and/or peroxisomal disorder. Given the obvious difficulties inherent to the clinical recognition of this disorder, the most practical route to diagnosis is 


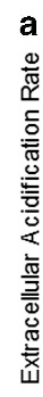

C

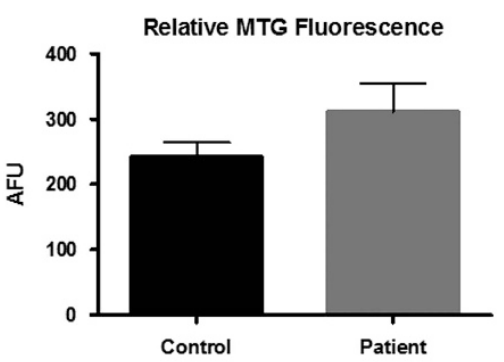

Glucose Stimulated ECAR

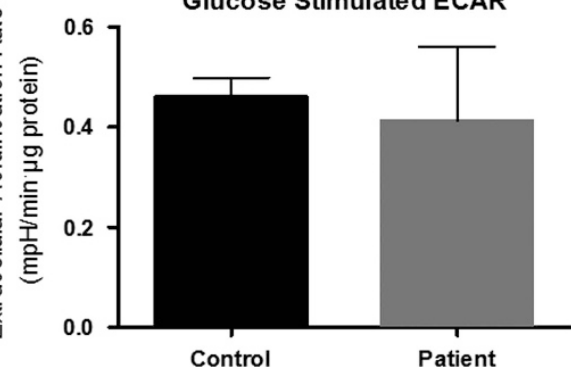

b

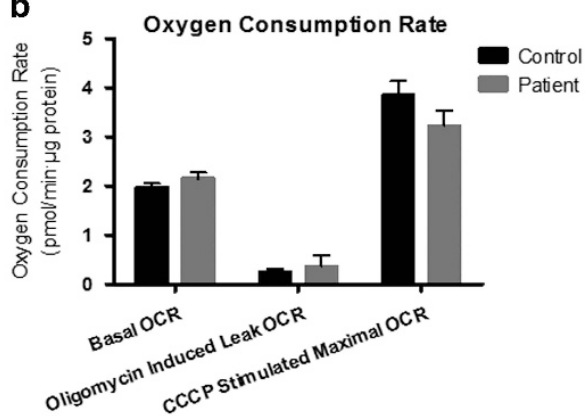

d

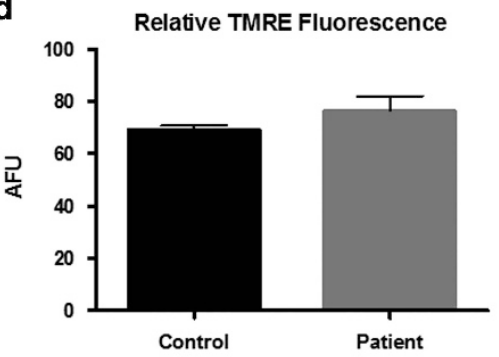

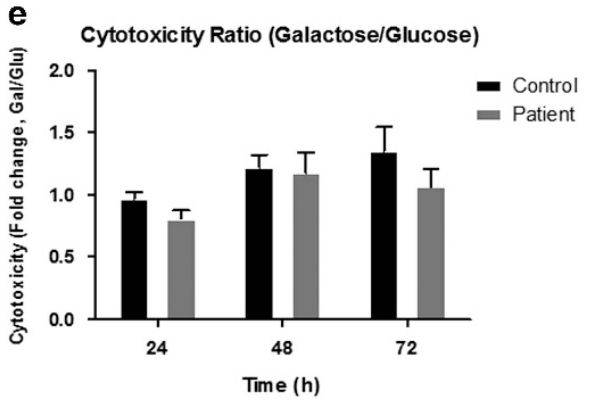

Figure 3 Bioenergetics, flow cytometry, and cell survival studies showed no differences in patient fibroblasts compared with control fibroblasts. (a) Bioenergetics - glucose-stimulated extracellular acidification rate (ECAR), reflecting reliance on glycolysis, was not different between patient and control fibroblasts. (b) Bioenergetics - oxygen consumption rate (OCR) was not different between patient and control fibroblasts. (c) Flow cytometry - fluorescence intensity of cells stained with MitoTracker Green FM (MTG) indicates no detectable difference in mitochondrial bulk. (d) Flow cytometry - fluorescence intensity of cells stained with tetramethylrhodamine ethyl ester (TMRE) indicates no identifiable difference in the mitochondrial membrane potential. (e) Cell survival - cytotoxicity ratios for cells grown in galactose (ie, under mandatory aerobic respiration) vs glucose (which permits ATP yield through glycolysis) were not different between patient and control cells ( $n=3$ for all panels; error bars represent mean \pm SEM; statistical significance tested using Student's $t$-test with $P<0.05$ ).

likely molecular testing as part of a panel or whole-exome/genome analysis with confirmation (where required) by confocal imaging of the mitochondrial network in patient cells.

We note that both the p.(Gly362Asp) substitution reported here and the p.Ala395Asp substitution reported by Waterham et al. ${ }^{10}$ are de novo missense substitutions of the middle domain of DMN1L, involved in homo-oligomerization of the protein. An immediately adjacent glycine-to-aspartate substitution, p.Gly363Asp, has been studied extensively in vitro, and this change appears to permit (normal) DNM1L tetramerization in the cytoplasm while abolishing higher-order self-assembly and polymerization-dependent GTPase activity. ${ }^{17}$ The phenotype in our proband is therefore most likely because of a dominant-negative effect owing to sequestration of wild-type DNM1L monomers in tetramers of reduced valency, that is, unable to participate in helical polymerization on mitochondrial membranes.

In contrast with the hyperlactatemia reported in the proband described by Waterham et al, ${ }^{10}$ we have yet to demonstrate a convincing respiratory phenotype in cells from our patient. How a generalized defect of mitochondrial and peroxisomal fission leads to a specific CNS phenotype is not clear, although one possibility may be that this is a disorder of mitochondrial distribution within neurons, analogous to the findings in the conditional Drpl substantia nigra knockout mouse. ${ }^{7}$ In these mice, loss of Drpl is associated with a dramatic decrease in mitochondrial mass, particularly in axons, where movement of the mitochondria became uncoordinated. ${ }^{7}$ The expression of DNM1L mRNA does appear to be highest in brain. ${ }^{5,18}$ Although the optimal therapeutic approach for this patient's condition is not known, the anomalous mitochondrial network architecture observed in patient cells could reasonably form the basis of an in vitro drug or genetic screen to select candidate agents for further study.

\section{CONFLICT OF INTEREST}

The authors declare no conflict of interest.

\section{ACKNOWLEDGEMENTS}

We acknowledge the participation of the proband and his family, without whom this work would not be possible. This work was supported by MitoCanada Foundation and was performed, in part, under the Care4Rare Canada Consortium funded by Genome Canada, the Canadian Institutes of 
Health Research (CIHR), the Ontario Genomics Institute, Ontario Research Fund, Genome Quebec, and CHEO Foundation. We thank Taila Hartley (Clinical Coordinator) and Chandree Beaulieu (Project Manager) at the Children's Hospital of Eastern Ontario (CHEO) Research Institute for their contribution to the infrastructure of Care4Rare. This work was selected for study by the Care4Rare (Enhanced Care for Rare Genetic Diseases in Canada) Consortium Gene Discovery Steering Committee: Kym Boycott (lead; University of Ottawa), Alex MacKenzie (co-lead; University of Ottawa), Jacek Majewski (McGill University), Michael Brudno (University of Toronto), Dennis Bulman (University of Ottawa), and David Dyment (University of Ottawa). We acknowledge the contribution of the high-throughput sequencing platform of the McGill University and Génome Québec Innovation Centre, Montréal, Canada. MT received a post-doctoral fellowship from the CIHR. GA received a Vanier Canada Graduate Scholarship. M-EH is funded by the CIHR (INMD; MOP 57810). DAD is supported by the CIHR Institute of Genetics Clinical Investigatorship Award.

1 Archer SL: Mitochondrial dynamics - mitochondrial fission and fusion in human diseases. N Engl J Med 2013; 369: 2236-2251.

2 Itoh K, Nakamura K, lijima M, Sesaki H: Mitochondrial dynamics in neurodegeneration. Trends Cell Biol 2013; 23: 64-71.

3 Labrousse AM, Zappaterra MD, Rube DA, van der Bliek AM: C. elegans dynamin-related protein DRP-1 controls severing of the mitochondrial outer membrane. Mol Cell 1999; 4: 815-826.

4 Smirnova E, Griparic L, Shurland DL, van der Bliek AM: Dynamin-related protein Drp1 is required for mitochondrial division in mammalian cells. Mol Biol Cell 2001; 12 : 2245-2256.
5 Smirnova E, Shurland D-L, Ryazantsev SN, van der Bliek AM: A human dynamin-related protein controls the distribuition of mitochondria. J Cell Biol 1998; 143: 351-358.

6 Wakabayashi J, Zhang Z, Wakabayashi $\mathrm{N}$ et al: The dynamin-related GTPase Drp1 is required for embryonic and brain development in mice. J Cell Biol 2009; 186: 805-816.

7 Berthet A, Margolis EB, Zhang J et al: Loss of mitochondrial fission depletes axonal mitochondria in midbrain dopamine neurons. J Neurosci 2014; 34: $14304-14317$.

8 Oettinghaus B, Schulz JM, Restelli LM et al: Synaptic dysfunction, memory deficits and hippocampal atrophy due to ablation of mitochondrial fission in adult forebrain neurons. Cell Death Differ 2016; 23: 18-28.

9 Shields LY, Kim H, Zhu L et al: Dynamin-related protein 1 is required for normal mitochondrial bioenergetic and synaptic function in CA1 hippocampal neurons. Cell Death Dis 2015; 6: e1725.

10 Waterham HR, Koster J, van Roermund CW, Mooyer PA, Wanders RJ, Leonard JV: A lethal defect of mitochondrial and peroxisomal fission. N Engl J Med 2007; 356: 1736-1741.

11 Yoon G, Malam Z, Paton T et al: G.0.6: Lethal disorder of mitochondrial fission caused by mutations in DNM1L. Neuromuscul Disord 2014; 24: 794, (abstract).

12 Liu H, Sawyer SL, Gos M et al: Atypical fibrodysplasia ossificans progressiva diagnosed by whole-exome sequencing. Am J Med Genet Part A 2015; 9999: 1-5.

13 Invernizzi F, D'Amato I, Jensen PB, Ravaglia S, Zeviani M, Tiranti V: Microscale oxygraphy reveals OXPHOS impairment in MRC mutant cells. Mitochondrion 2012; 12: 328-335.

14 Adzhubei IA, Schmidt S, Peshkin L et al: A method and server for predicting damaging missense mutations. Nat Methods 2010; 7: 248-249.

15 Exome Aggregation Consortium [http://exac. broadinstitute.org] (accessed August 2015).

16 Kumar P, Henikoff S, Ng PC: Predicting the effects of coding non-synonymous variants on protein function using the SIFT algorithm. Nat Protoc 2009; 4: 1073-1081.

17 Chang CR, Manlandro CM, Arnoult D et al: A lethal de novo mutation in the middle domain of the dynamin-related GTPase Drp1 impairs higher order assembly and mitochondrial division. J Biol Chem 2010; 285: 32494-32503.

18 Imoto M, Tachibana I, Urrutia R: Identification and functional characterization of a novel human protein highly related to the yeast dynamin-like GTPase Vps1p. J Cell Sci 1998; 111: 1341-1349.

Supplementary Information accompanies this paper on European Journal of Human Genetics website (http://www.nature.com/ejhg) 